\title{
Charm and beauty physics at ATLAS
}

\author{
Takashi Matsushita* Kobe University \\ E-mail: Takashi.Matsushitalcern.ch
}

\section{On behalf of the ATLAS Collaboration}

ATLAS has a rich charmonium and beauty physics programme. After a few $\mathrm{pb}^{-1}$ of $7 \mathrm{TeV}$ collision data have been taken at the LHC, ATLAS will be able to start probing the new energy regime with decays of the $\psi$ and $\Upsilon$ families of mesons into pairs of muons. The very first measurements, possible with only a few $\mathrm{pb}^{-1}$ of data, will include the fraction of $J / \psi$ mesons produced in $B$-hadron decays, the average lifetime of $B$-hadrons decaying via $J / \psi$, and the differential $J / \psi$ production cross section. Crucial to all of these measurements are the performance of the muon triggers and the offline muon reconstruction and identification, as well as the inner detector performance and tracking. The performance of these elements with respect to the LHC data taken thus far as well as the prospects for the early charm and beauty physics at ATLAS are presented.

XVIII International Workshop on Deep-Inelastic Scattering and Related Subjects, DIS 2010 April 19-23, 2010

Firenze, Italy

\footnotetext{
* Speaker.
} 


\section{Introduction}

ATLAS [1] is a multi-purpose detector operating at the Large Hadron Collider (LHC) located at CERN. The LHC has been designed to provide collisions of protons at a centre-of-mass energy of $14 \mathrm{TeV}$ with luminosity of $10^{34} \mathrm{~cm}^{-2} \mathrm{~s}^{-1}$. One of the first physics results of ATLAS will be an analysis of $J / \psi$ and $\Upsilon$ productions decaying into pairs of muons. Measuring the $J / \psi$ production and its properties is a crucial step for performing studies on various $B$-meson decay channels. The narrow quarkonia resonances will be used to study trigger performance, muon reconstruction efficiency, detector alignment, material effects and magnetic field.

There are various models for the production mechanism of quarkonium, including colouroctet model (COM) and colour-singlet model [2]. The COM describes the inclusive quarkonium cross-section at the Tevatron, however, the model does not describe the polarisation [3]. The COM predicts that the prompt quarkonia produced in $p p$ collisions are transversely polarised, with the polarisation increasing as a function of the transverse momentum $\left(p_{T}\right)$. Other models predict different $p_{T}$ dependencies of the polarisation. ATLAS has prepared analysis for probing the region of large $p_{T}$ accessible at the LHC with high statistics, in order to improve an understanding of the production mechanism.

The prospects for the early charm and beauty physics at ATLAS are described in this paper. Some of the studies performed with Monte Carlo samples with $14 \mathrm{TeV}$ centre-of-mass energy are reviewed in the following sections. In addition, results of performance study on detector elements relevant to the analysis, which has been carried out with the data taken at a centre-of-mass energy of $900 \mathrm{GeV}$ in 2009, are presented.

\section{The ATLAS detector}

For the charm and beauty physics presented in the following sections, the Inner Detector tracking devices (ID), the Muon Spectrometer (MS) and the trigger system are of particular importance.

The ID consists of a pixel detector, a silicon strip detector and a Transition Radiation Tracker (TRT). The ID is located inside magnetic field of 2 Tesla and covers central pseudo-rapidity ( $\eta$ ) region, $|\eta|<2.5$. The ID barrel (end-cap) parts consists of 3 (2x3) pixel layers, 4 (2x9) layers of double-sided silicon strip modules, and 73 (2x160) layers of TRT straws. Fig. 1(a) shows invariant mass distribution of the $K_{s}^{0}$ candidates collected in 2009. The mean and resolution of the mass peak obtained from the fit in data is consistent with simulation and with the PDG value for the mass. The agreement demonstrates accuracy of the track momentum scale and excellent modelling of the ID's 2 T solenoid magnetic field [4].

The MS has been designed to detect muons, to measure their momentum using the toroidal magnetic field and to issue muon triggers. The MS covers $|\eta|<2.7$ with precision tracking chambers and $|\eta|<2.4$ for triggering with fast response chambers. The chambers are arranged in three layers, so that high- $p_{T}$ particles traverse at least three stations with a lever arm of several metres. Fig. 1(b)] shows $p_{T}$ distribution of offline reconstructed muons with data collected in 2009. The data agree with the Monte Carlo prediction within the statistical errors of the data [4].

ATLAS employs three level trigger system to reduce the output event rate down to about $200 \mathrm{~Hz}$ from an initial LHC bunch-crossing rate of $40 \mathrm{MHz}$. The level1 trigger is based on a hardware trig- 


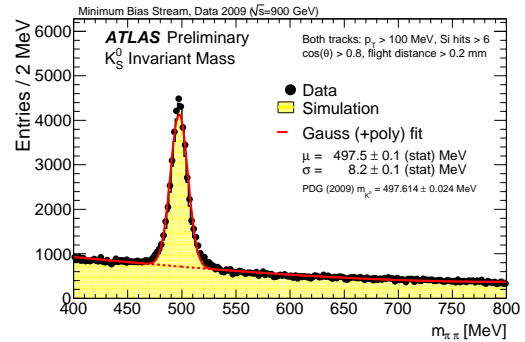

(a) $K_{s}^{0}$ mass spectrum. Dots are data and the histogram shows Monte Carlo simulation.

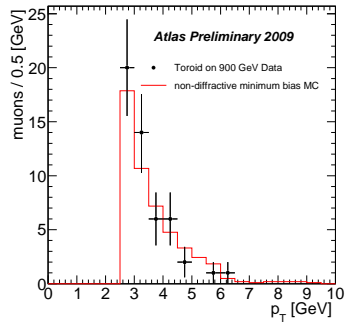

(b) $p_{T}$ distribution of reconstructed muons. Dots are data and the histogram shows Monte Carlo simulation.

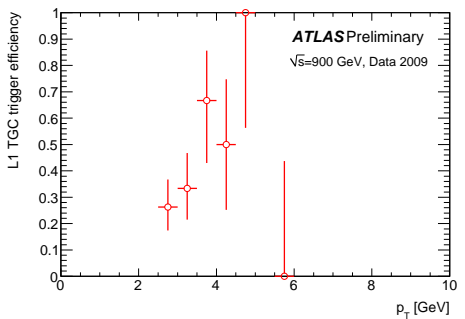

(c) Level1 muon trigger efficiency with respect to reconstructed muons in the end-cap regions, $1.05<|\eta|<2.4$.

Figure 1: Detector performance plots with data collected at a centre-of-mass energy of $900 \mathrm{GeV}$.

ger with calorimeter and muon information to reduce the event rate to $75 \mathrm{kHz}$. The level 2 trigger is a software trigger used to confirm and refine the levell trigger decisions, reducing the event rate to $2 \mathrm{kHz}$. The Event Filter (EF) performs event selection using more complex algorithms and reduces the event rate to $200 \mathrm{~Hz}$.

The level1 muon trigger searches for patterns of hits within $|\eta|<2.4$ consistent with high$p_{T}$ muons originating from the interaction region. Dedicated trigger for the charm and beauty physics have been developed in ATLAS for the level2 and the EF, which is based on single-muon and di-muon level1 triggers with low- $p_{T}$ thresholds [6]. At level2, a fast muon reconstruction using precision muon tracking chambers combined with ID measurements allow early rejection of muons from decay-in-flight. At the EF, full event data as well as alignment and calibration data are used to refine the trigger decision using offline-like algorithms. Fig. 1(c) shows level1 muon trigger efficiency with respect to offline reconstructed muons in the end-cap regions, $1.05<|\eta|<2.4$ 《5]. The level1 muon trigger is operational and working, although the very limited statistics available do not allow for any quantitative estimate of trigger efficiencies.

\section{Expected results with Monte Carlo study}

The measurement of prompt $J / \psi$ and $\Upsilon$ cross-sections as well as exclusive $b$-quark production cross-section are planned for the early running period of ATLAS. The following results have been obtained with Monte Carlo study with $14 \mathrm{TeV}$ centre-of-mass energy [6].

\subsection{Heavy prompt quarkonia}

For the prompt $J / \psi$ and $\Upsilon$ production cross-sections measurement, events are seeded by a level1 di-muon trigger with 4 and $6 \mathrm{GeV}$ thresholds (mu4mu6) for the two muons. Both muons are required to come from the primary vertex with a pseudo-proper time of less than $0.2 \mathrm{ps}$ at offline analysis. Fig. 2(a) shows invariant mass distribution of the quarkonium signal and main background. Peaks from the $J / \psi$ and $\Upsilon$ are clearly seen. With $1 \mathrm{pb}^{-1}$ of data, about 15000 $J / \psi$ and $2500 \Upsilon$ are expected within mass windows of $m_{J / \psi}^{P D G} \pm 300 \mathrm{MeV}$ and $m_{\Upsilon}^{P D G} \pm 1 \mathrm{GeV}$, respectively. The expected signal to noise ratios are 60 for $J / \psi$ and 10 for $\Upsilon$. With $10 \mathrm{pb}^{-1}$ of 


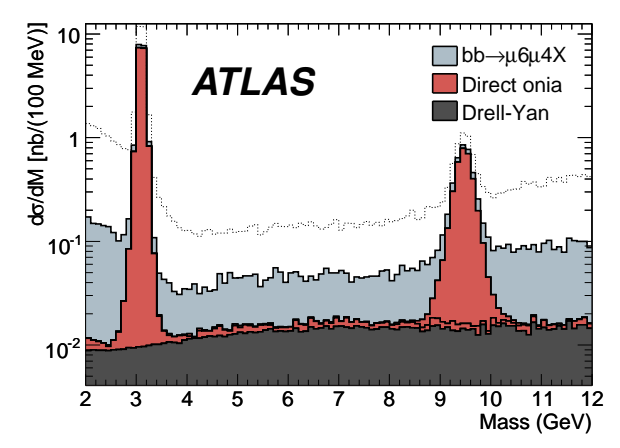

(a) Invariant mass of di-muons, selected with a dimuon trigger. The dotted line shows the cumulative distribution without vertex and pseudo-proper time cuts.
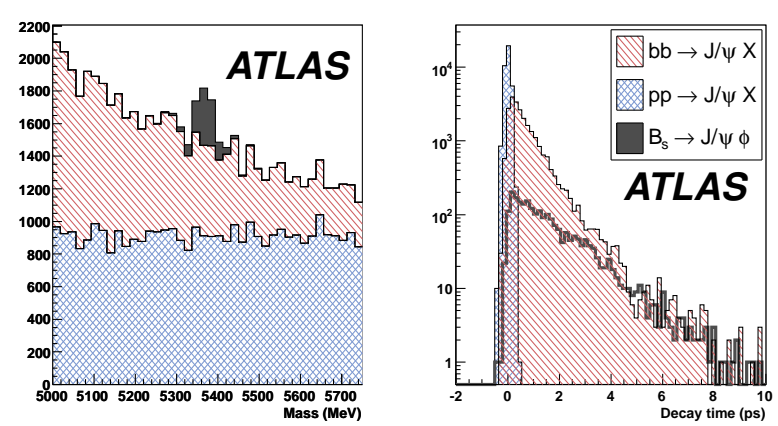

(b) The distributions of the reconstructed $B_{S}^{0}$ mass and decay time expected with $150 \mathrm{pb}^{-1}$.

Figure 2: Invariant mass distribution of di-muons and $B_{s}^{0}$ distributions.

data, measurement accuracy on differential cross-section in terms of $p_{T}$ is expected to be an order of $1 \%$ and $5 \%$ for $J / \psi$ and $\Upsilon$, respectively.

\subsection{Polarisation measurements}

The polarisation of the $J / \psi$ and $\Upsilon$ states can be obtained by measuring the angular distribution of the muons from the $J / \psi$ and $\Upsilon$ decays. The polarisation angle, $\theta^{*}$, is defined as the angle between the $\mu^{+}$and the $J / \psi(\Upsilon)$ momentum in the rest frame of the $J / \psi(\Upsilon)$. The angular distribution is related to the polarisation parameter, $\alpha$, through the formula, $d N / d \cos \theta^{*} \propto$ $\left(1+\alpha \cos ^{2} \theta^{*}\right)$. The polarisation parameter is $+1,-1$ and 0 for transversely polarised, longitudinally polarised and unpolarised production, respectively. In order to increase the sensitivity to the polarisation, single-muon trigger with a threshold of $10 \mathrm{GeV}$ (mu10) is used to select di-muon at offline analysis, in addition to mu4mu6 trigger based di-muon selection described above. For the mu10 trigger based selection, a track with $p_{T}>0.5 \mathrm{GeV}$ is required in a cone around the triggered muon direction, demanding both tracks originating from the primary vertex. Here cone is defined as $\Delta R_{\mu, \text { track }}=\left(\Delta \phi_{\mu, \text { track }}^{2}+\Delta \eta_{\mu, \text { track }}^{2}\right)^{1 / 2}$ and cone size of 3 is used for the selection.

With $10 \mathrm{pb}^{-1}$ of data, an estimated precision on $\alpha$ is an order of $0.02-0.06$ and 0.2 for $\alpha(J / \psi)$ and $\alpha(\Upsilon)$, respectively, in a momentum range of $9-21 \mathrm{GeV}$ and above.

\section{$3.3 B^{+} \rightarrow J / \psi K^{+}$}

Owing to the large production rate and its clear topology, $B^{+} \rightarrow J / \psi K^{+}$will be one of the first fully-reconstructed $B$ decays at ATLAS. The channel can serve as a reference for rare $B$ decay searches, whose cross-section will be measured relative to its cross-section, thus allowing the cancellation of common systematic uncertainties. Furthermore, the channel can be used for flavour tagging algorithm calibrations. The studies on ID calibration and alignment will be performed with the well known properties of $B^{+}$. 
The event selection is based on a mu4mu6 trigger. In order to reconstruct the $J / \psi$, cuts are applied on two-track vertex and on the invariant mass. An additional track which is displaced from the primary vertex is required for the $K^{+}$. Further cuts on the common vertex of the three particles are then applied. About 1600 signal events are expected for $10 \mathrm{pb}^{-1}$ of data. The differential crosssection, $d \sigma / d p_{T}$, is expected to be measured with a statistical precision of $10 \%$. In addition, the lifetime of $B^{+}$is expected to be measured to a statistical precision of $2.5 \%$.

3.4 $B_{d}^{0} \rightarrow J / \psi K^{0 *}$ and $B_{s}^{0} \rightarrow J / \psi \phi$

The decays, $B_{d}^{0} \rightarrow J / \psi K^{0 *}$ and $B_{s}^{0} \rightarrow J / \psi \phi$, are promising channels for the startup of LHC. Precise measurements of the parameters from the two weak eigenstates in the $B_{s}^{0}-\overline{B_{s}^{0}}$ system such as $\Delta m$ and $\Delta \Gamma$ will be valuable inputs for improving our knowledge on this sector.

First, the $B_{d}^{0}\left(B_{s}^{0}\right)$ are selected with a mu4mu6 trigger. Second, the $J / \psi$ and $K^{0 *}(\phi)$ are reconstructed from two muons and from two tracks assuming a kaon and a pion (two kaons), respectively. Then cuts on vertex and $p_{T}$ are applied. The invariant mass and decay time distributions for the reconstructed $B_{s}^{0}$ events are shown in Fig. 2(b), About 1000 candidates are expected for $B_{d}^{0}$ and $B_{s}^{0}$ with an integrated luminosity of $10 \mathrm{pb}^{-1}$ and $150 \mathrm{pb}^{-1}$, respectively. By performing a simultaneous fit on the invariant mass and decay time, statistical precisions of $10 \%$ on the lifetimes of $B_{s}^{0}$ and $B_{d}^{0}$ can be achieved at the early stage.

\section{Summary}

The LHC has started its operation in 2009. The performances of the detector components relevant for the analysis presented in this paper have been studied with collision data collected at $900 \mathrm{GeV}$ centre-of-mass energy. ATLAS is well prepared to perform study on charm and beauty physics. Cross-section ratio of indirect to prompt $J / \psi$ productions will be measured with a few $\mathrm{pb}^{-1}$ of data collected at a $7 \mathrm{TeV}$ centre-of-mass energy. With an order of $100 \mathrm{pb}^{-1}$ of data, polarisation measurement will be possible which can access an interesting high $p_{T}$ region. Cross-sections and lifetime measurements with exclusive decays of $B$ mesons, $B^{+} \rightarrow J / \psi K^{+}$, $B_{d}^{0} \rightarrow J / \psi K^{0 *}$ and $B_{s}^{0} \rightarrow J / \psi \phi$, will be possible with an integrated luminosity of $10-150 \mathrm{pb}^{-1}$.

\section{References}

[1] ATLAS Collaboration, JINST 3, S08003 (2008)

[2] G. T. Bodwin, E. Braaten, G. P. Lepage, Phys. Rev. D 51, 1125 (1995); ibid D 55, 5853 (1997); N. Brambilla et al., CERN Yellow Report, CERN-2005-005, 2005

[3] CDF Collaboration, Phys. Rev. Lett. 99, 132001 (2007)

[4] ATLAS Collaboration, e-Print: arXiv:1005.5254 (2010)

[5] ATLAS Collaboration, ATLAS-CONF-2010-013

[6] ATLAS Collaboration, CERN-OPEN-2008-020; E. Etzion, J. Ginzburg Nucl. Phys. B 187, 136 (2009) 